\section{Extended $M$-sequence codes for SAC FO-CDMA PONs applications}

\section{S.-P. Tseng and J. Wu}

A new code structure, namely extended $M$-sequence (EMS) codes, is presented suitable for passive optical networks (PON). Based on these codes, a spectral-amplitude-coding (SAC) fibre-optic codedivision multiple-access system (FO-CDMA) is designed with shared optical line terminal (OLT) and flexible optical network units (ONUs) for PON applications. The results shows the proposed system has excellent performance.

Introduction: The spectral-amplitude-coding (SAC) fibre-optic code division multiple-access system (FO-CDMA) technique with fixed inphase cross-correlation has received a lot of attention since the influence of the multiple access interference (MAI) can be eliminated by using the balanced detector $[1-5]$. On the other hand, the array waveguide grating (AWG) was proposed as a codec device for the compact SAC FOCDMA system with $M$-sequence (MS) codes [5]. However, the decoder design is not practical for FO-CDMA passive optical networks $(\mathrm{PON})$ applications since the optical networks units (ONUs) may be in many different locations. Moreover, MS codes have very restrictive code lengths and are unable to provide many users in the optical network simultaneously owing to their cross-correlation being approximately equal to one-quarter of the code length. In consideration of the above views, we propose a new code family, namely EMS code, to relax the code length restriction and increase the number of simultaneous users. We employ these codes to design a compact optical line terminal (OLT) and flexible ONUs for PON applications. The performance of the proposed system is analysed with the consideration of phase-induced intensity noise (PIIN) and thermal noise. The results show that EMS codes can accommodate more simultaneous users and still retain excellent properties. Moreover, the new code family is flexible in code length.

Code designs: EMS codes can be constructed by the following steps. First, we denote $\boldsymbol{Y}_{k}=\left(y_{k}, 0, y_{k 1}, \ldots, y_{k, M-1}\right)=\mathrm{T}^{k} \boldsymbol{Y}_{0}(k=0,1, \ldots$, $M-1)$ as the MS codeword of length $M$, where $\mathrm{T}^{k}$ is an operator designed to shift codes cyclically to the right $k$ times and $\boldsymbol{Y}_{0}$ is the original code. Next, we construct the EMS code $\boldsymbol{X}_{k, l}$ based on the MS code by using the following mapping:

$$
\begin{aligned}
\boldsymbol{M} & =\left|\boldsymbol{X}_{k, l}\right|_{W \times W}=\left[x_{k, l}(w)\right]=\operatorname{kron}\left(\left|\boldsymbol{Y}_{k}\right|_{M \times M}, I_{L}\right) \\
& =\left|\begin{array}{ccc}
y_{0,0} * I_{L} & \cdots & y_{0, M-1} * I_{L} \\
\vdots & \ddots & \vdots \\
y_{M-1,0} * I_{L} & \cdots & y_{M-1, M-1} * I_{L}
\end{array}\right|_{M L \times M L}
\end{aligned}
$$$$
k=0,1, \ldots, M-1, l=0,1, \ldots, L-1 \text { and } w=0,1, \ldots, M L-1
$$

and

$$
\overline{\boldsymbol{M}}=\left|\overline{\boldsymbol{X}}_{k, l}\right|_{W \times W}=\left[\bar{x}_{k, l}(w)\right]=\operatorname{kron}\left(\left|\overline{\boldsymbol{Y}}_{k}\right|_{W \times W}, I_{L}\right)
$$

where $x_{k, l}(w)$ is the $w$ th element of $\boldsymbol{X}_{k, l}$, kron $(\cdot)$ is a Kronecker product matrix, $\overline{\boldsymbol{Y}}_{k}=\left(1-y_{k, 0}, 1-y_{k, 1}, \ldots, 1-y_{k, M-1}\right)$ is the complementary of $\boldsymbol{Y}_{k},{ }^{*}$ is multiplication, and $I_{L}$ is the $L$-by- $L$ identity matrix. Here, $L$ represents the number of groups and is a positive integer. Thus, the code length is $M L$. Note that $\boldsymbol{X}_{k, l}$ and $\overline{\boldsymbol{X}}_{k, l}$, are, respectively, the $(k L+$ $l+1)$ th rows of $\boldsymbol{M}$ and $\overline{\boldsymbol{M}}$. Table 1 shows examples of $\boldsymbol{X}_{k, l}$ and $\overline{\boldsymbol{X}}_{k, l}$, for $M=3$ and $L=3$.

Table 1: EMS codewords for $M=3$ and $L=3$

\begin{tabular}{|c|c|c|c|}
\hline$k$ & $l$ & Codeword $\mathrm{X}_{k, l}$ & Codeword $\overline{\mathrm{X}}_{k, l}$ \\
\hline 0 & 0 & 100100000 & 000000100 \\
\hline 0 & 1 & 010010000 & 000000010 \\
\hline 0 & 2 & 001001000 & 000000001 \\
\hline 1 & 0 & 000100100 & 100000000 \\
\hline 1 & 1 & 000010010 & 010000000 \\
\hline 1 & 2 & 000001001 & 001000000 \\
\hline 2 & 0 & 100000100 & 000100000 \\
\hline 2 & 1 & 010000010 & 000010000 \\
\hline 2 & 2 & 001000001 & 000001000 \\
\hline
\end{tabular}

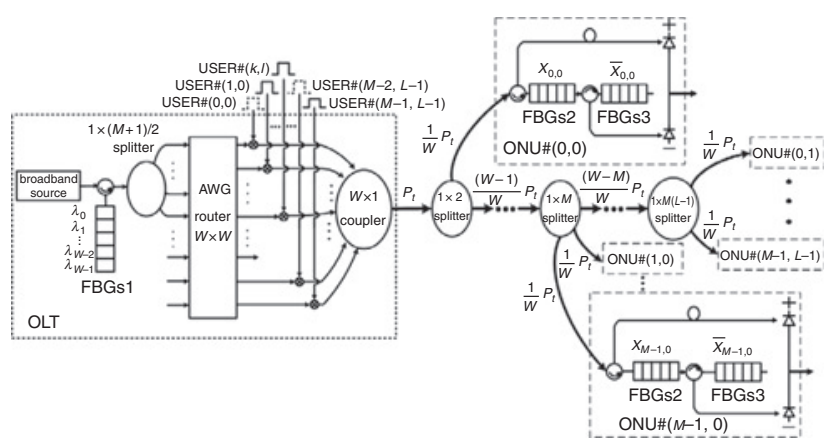

Fig. 1 Proposed SAC FO-CDMA PON with EMS codes

System description: Fig. 1 shows the proposed SAC FO-CDMA PON, which includes three parts: one OLT at the central office, several optical splitters, and $M L$ ONUs. The EMS code, $\boldsymbol{X}_{k, l}$, is assigned as the $(k, l)$ th ONU codeword. A compact OLT consists of an unpolarised broadband light source, an optical circulator, one set of fibre Bragg gratings (FBGs), FBGs1, with the corresponding chips of $\lambda_{0}, \lambda_{1}, \ldots, \lambda_{W-1}$, an optical splitter, one AWG router, $M L$ electro-optic modulators (EOM), and an optical coupler. The light source has flat power spectral density (PSD) and the bandwidth of the FBG is the same as those of the wavelength channels of the AWG router. For a system with a total of $W$ ONUs, we can have an equal value of power at all ONUs, as shown in Fig. 1, if the splitting ratio is carefully designed. Moreover, we can adjust the splitting ratio or use optical attenuators if the fibre loss must be taken into consideration. Each of the ONUs is composed of two optical circulators, FBGs2, FBGs3, one balanced photodetector, and a delay line for compensating the delay caused by FBGs 2 and FBGs3. The ordinals of FBGs2 and FBGs3 are arranged as the inverse ordinal of FBGs1 to compensate for the different round-trip delays. When the optical signal enters FBGs1, the corresponding chips matched to one free spectral range (FSR) of the AWG router are reflected back and broadcast to the input ports of the AWG router via a $1 \times(N+1) / 2$ splitter according to the cardinal of code weight of $\boldsymbol{X}_{0,0}$. Then, these reflected signals input to the different input ports of the AWG router are directed to the appropriate output ports of the AWG router in accordance with

$$
\text { \#input port }+ \text { \#output port }(\bmod W)=\# \text { wavelength }
$$

where mod $W$ denotes modulo- $W$ addition. Based on the cyclic property of the AWG router, the $(w+k L+l(\bmod W))$ th chip of $\boldsymbol{X}_{k, l}$ is obtained from the $w$ th input port of AWG router and appears at the $(k L+l)$ th output port of the AWG router $[4,5]$. Therefore, we can find that $M L$ EMS codewords appear at different output ports of the AWG router simultaneously. After the encoding process, the signals $\boldsymbol{S}$ are summed in the $W \times 1$ coupler and can be written as

$$
\boldsymbol{S}=\left(s_{0}, s_{1}, \ldots, s_{W-1}\right)=\sum_{k=0}^{M-1} \sum_{l=0}^{L-1} b_{k, l} \boldsymbol{X}_{k, l}
$$

where $b_{k, l}$ is the binary data bit of the $(k, l)$ th user. Assume that the $(j, s)$ th user is the desired user. The correlation $(\theta)$ between $\boldsymbol{X}_{k, l}$ and $\boldsymbol{X}_{j, s}$ is given by

$$
\begin{aligned}
\theta_{\mathrm{xx}}(j, s, k, l) & =\sum_{w=0}^{W-1} x_{j, s}(w) x_{k, l}(w) \\
& =\left\{\begin{array}{rll}
(M+1) / 2, & \text { if } & s=l, j=k \\
(M+1) / 4, & \text { if } & s=l, j \neq k \\
0, & \text { if } s \neq l
\end{array}\right.
\end{aligned}
$$

Because FBGs2 and FBGs3 of the $(j, s)$ th ONU correspond to $\boldsymbol{X}_{j, s}$ and $\overline{\boldsymbol{X}}_{j, s}$, the correlation subtraction at the $(j, s)$ th ONU is given by

$$
\begin{aligned}
& \boldsymbol{Z}=\theta_{\mathrm{xx}}(j, s, k, l)-\theta_{\mathrm{x} \overline{\mathrm{x}}}(j, s, k, l) \\
& =\left\{\begin{array}{rrr}
(M+1) / 2, & \text { if } & s=l \text { and } j=k \\
0, & \text { otherwise }
\end{array}\right.
\end{aligned}
$$

It is obvious that the MAI is eliminated at this moment. If the interference user belongs to a different group, the corresponding chips will pass through two sets of FBGs without giving rise to PIIN in the 
photodetectors. Moreover, the complexity of the ONU is reduced without attenuators.

System performance: In the following analysis, PIIN [1] and thermal noise are considered [3, 4]. Assume that a light source has the optical bandwidth $\Delta v$ and magnitude $P_{t} / \Delta v$, where $P_{t}$ represents the effective power of the light source at the output of the $W \times 1$ coupler. Note that $P_{t r}$ represents the optical power of the light source at the input of each ONU and equals $P_{t} / W . P_{t r}$ is also proportional to the value of $\boldsymbol{S}$ expressed in (3). Using (3), (4) and (5), we obtain the signal-to-noise ratio $(\mathrm{SNR})$ as $[1,3,4]$

$$
\begin{aligned}
\mathrm{SNR}= & \frac{\boldsymbol{P}_{\text {sig }}}{\left\langle i^{2}\right\rangle} \\
= & \frac{\left(R P_{t r}(M+1) / 2 M L\right)^{2}}{\left(B R^{2} P_{t r}^{2} / 2 \Delta v M L\right)\{((M+1) / 2)+\lfloor K / L\rfloor} \\
& {\left.\left[(M+1)+\left((M+1)^{2} / 4 M\right)(\lfloor(K / L)\rfloor-1)\right]\right\}+4 K_{b} T_{n} B l R_{L} }
\end{aligned}
$$

where $K$ is the number of active users, \lfloor\rfloor is the floor function, $\boldsymbol{P}_{\text {sig }}$ is the power of average photocurrent and $\left\langle i^{2}\right\rangle$ is the variance of total noise photocurrent. For comparison, we take the simulation parameters listed in Table 2 from [3]. Based on the Gaussian approximation, i.e. $\mathrm{BER}=1 / 2 \times \operatorname{erfc}(\mathrm{SNR} / 8)^{1 / 2}$, we can compute the BER against the number of active users for given codes, as shown in Fig. 2. It is seen that the number of active users for EMS code is larger than for modified quadratic congruence (MQC) code, complementary Walsh-Hadamard $(\mathrm{CWH})$ code, and MS code. In addition, as $L$ increases, EMS code can provide a lower BER under the condition of having similar code length.

Table 2: Typical parameters used in calculation

\begin{tabular}{|c|c|}
\hline Power of light source at each ONU $\left(P_{t r}\right)$ & $-10 \mathrm{dBm}(100 \mu \mathrm{W})$ \\
\hline PD responsivity for InGaAs at $1550 \mathrm{~nm}(R)$ & $0.93 \mathrm{~A} / \mathrm{W}$ \\
\hline Noise-equivalent electrical bandwidth $(B)$ & $320 \mathrm{MHz}$ \\
\hline Boltzmann's constant $\left(K_{b}\right)$ & $1.38 \times 10^{-23} \mathrm{~J} /{ }^{\circ} \mathrm{K}$ \\
\hline Absolute temperature $\left(T_{n}\right)$ & $300^{\circ} \mathrm{K}$ \\
\hline Load resistor $\left(R_{\mathrm{L}}\right)$ & $1030 \Omega$ \\
\hline Spectral widths of light source $(\Delta v)$ & $30 \mathrm{~nm}$ \\
\hline Data bit rate $\left(R_{b}\right)$ & $622 \mathrm{Mbit} / \mathrm{s}$ \\
\hline
\end{tabular}

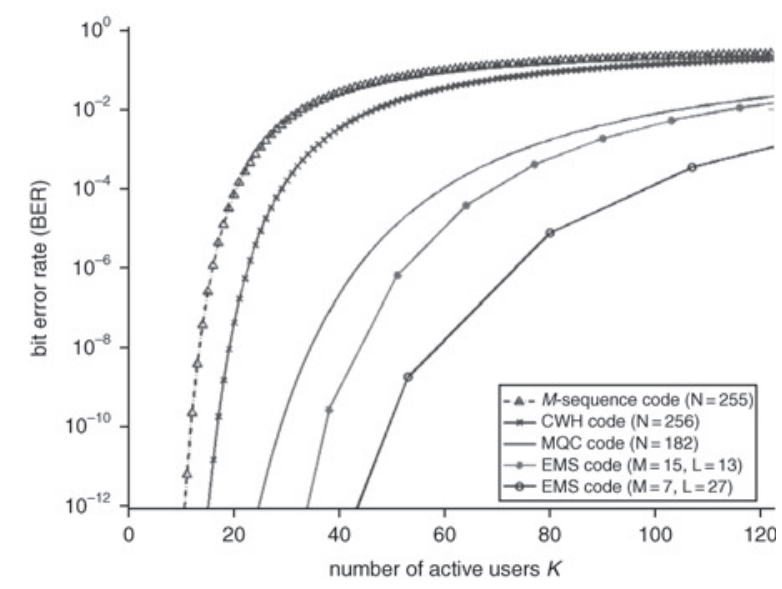

Fig. 2 BER against number of active users
Conclusions: A new code family is proposed, namely EMS codes, for SAC FO-CDMA PON applications. A shared OLT at the central office and simple ONUs are designed to eliminate MAI. The numerical results show that the proposed SAC FO-CDMA PON can accommodate a large number of active users.

Acknowledgment: This work was supported in part by Ministry of Education and Nation Science Council, Taiwan, under Grants MSE 96R0062-AE00-04 and NSC 96-2221-E-002-084.

(C) The Institution of Engineering and Technology 2008

16 November 2007

Electronics Letters online no: 20083321

doi: 10.1049/el:20083321

S.-P. Tseng and J. Wu (Institute of Communication Engineering and Department of Electrical Engineering, National Taiwan University, Taipei, Taiwan 106, Republic of China)

E-mail: wujsh@cc.ee.ntu.edu.tw

\section{References}

1 Smith, E.D.J., Blaikie, R.J., and Taylor, D.P.: 'Performance enhancement of spectral-amplitude-coding optical CDMA using pulse-position modulation', IEEE Trans. Commun., 1998, 46, pp. 1176-1185

2 Huang, J.F., and Hsu, D.Z.: 'Fiber-grating-based optical CDMA spectral coding with nearly orthogonal m-sequence codes', IEEE Photonics Technol. Lett., 2000, 12, pp. 1252-1254

3 Wei, Z., Shalaby, H.M.H., and Ghafouri-Shiraz, H.: 'Modified quadratic congruence codes for fiber Bragg-grating-based spectral-amplitudecoding optical CDMA systems', J. Lightwave Technol., 2001, 19, pp. $1274-1281$

4 Yang, C.C.: 'Compact bi-direction optical CDMA encoder/decoder with low power dissipation', Optical Fibre Technol., 2007, 13, pp. 254-259

5 Yang, C.C., Huang, J.F., and Tseng, S.P.: 'Optical CDMA network codecs structured with $\mathrm{M}$-sequence codes over waveguide-grating routers', IEEE Photonics Technol. Lett., 2004, 16, pp. 641-643 